\title{
Compact Minimal $C R$ Submanifolds of a Complex Projective Space with Positive Ricci Curvature
}

\author{
Mayuko KON \\ Hokkaido University \\ (Communicated by M. Guest)
}

\begin{abstract}
We give a reduction theorem for the codimension of a compact $n$-dimensional minimal proper $C R$ submanifold $M$ immersed in a complex projective space $C P^{m}$ with complex structure $J$, under the assumption that the Ricci curvature of $M$ is equal to or greater than $n-1$. Moreover, we classify compact $n$-dimensional minimal $C R$ submanifolds whose Ricci tensor $S$ satisfies $S(X, X) \geq(n-1) g(X, X)+k g(P X, P X), k=0,1,2$, for any vector field $X$ tangent to $M$, where $P X$ is the tangential part of $J X$.
\end{abstract}

\section{Introduction}

The purpose of the present paper is to study the pinching problem in terms of Ricci curvatures of minimal $C R$ submanifolds immersed in a complex projective space.

Let $C P^{m}$ denote the complex projective space of real dimension $2 m$ (complex dimension $m$ ) with constant holomorphic sectional curvature 4 and Kähler structure $(J, g)$. Let $M$ be a real $n$-dimensional Riemannian manifold isometrically immersed in $C P^{m}$ with induced metric $g$. If there exist a differentiable holomorphic distribution $H: x \mapsto H_{x} \subset T_{x}(M)$ and complementary orthogonal anti-invariant distribution $H^{\perp}$, then $M$ is called a $C R$ submanifold. In particular, when $M$ satisfies $J T_{x}(M)^{\perp} \subset T_{x}(M)$ for any point $x$ of $M, M$ is called a generic submanifold. Any real hypersurface is obviously generic.

In [8], Kon proved that if the Ricci tensor $S$ of a compact $n$-dimensional minimal $C R$ submanifold $M$ of $C P^{m}$ satisfies $S(X, X) \geq(n-1) g(X, X)+2 g(P X, P X)$, then $M$ is a real projective space $R P^{n}$, or a complex projective space $C P^{n / 2}$, or a pseudo-Einstein real hypersurface $\pi\left(S^{k}(1 / \sqrt{2}) \times S^{k}(1 / \sqrt{2})\right)(k=(n+1) / 2)$ of some $C P^{(n+1) / 2}$ in $C P^{m}$, where $S^{k}(r)$ is a $k$-dimensional sphere of radius $r, \pi$ is the Hopf fibration and $P X$ is the tangential part of $J X$ (see also [7]). For a minimal real hypersurface $M$ of $C P^{m}(m \geq 3)$, Maeda [9] studied the pinching problem in terms of Ricci curvatures of $M$. He proved that if the Ricci tensor $S$ of a minimal real hypersurface satisfies $(2 m-2) g(X, X) \leq S(X, X) \leq 2 m g(X, X)$, then it is locally congruent to $\pi\left(S^{m}(1 / \sqrt{2}) \times S^{m}(1 / \sqrt{2})\right)$.

Received September 18, 2007; revised June 29, 2009; revised July 20, 2010 Mathematics Subject Classification: 53C40, 53C55

Key words and phrases: Ricci curvature, $C R$ submanifold, complex projective space 
On the other hand, Yamagata-Kon [14] proved that if the Ricci tensor $S$ of a compact $n$-dimensional minimal generic submanifold $M$ of $C P^{m}$, which is not totally real, satisfies $S(X, X) \geq(n-1) g(X, X)$, then $M$ is a real hypersurface of $C P^{m}$, that is, $2 m-n=1$.

In this paper, we prove a reduction theorem for the codimension of a compact $n$ dimensional minimal proper $C R$ submanifold $M$ in $C P^{m}$. We prove that if the Ricci curvature of $M$ is equal to or greater than $n-1$, then $M$ is a real hypersurface of some $C P^{(n+1) / 2}$ in $C P^{m}$ (Theorem 2 ). Using this result, we classify compact $n$-dimensional minimal $C R$ submanifolds $M$ immersed in $C P^{m}$ whose Ricci tensors $S$ satisfy $S(X, X) \geq$ $(n-1) g(X, X)+k g(P X, P X), k=0,1,2$, for any vector field $X$ tangent to $M$ (Theorem $3,4,5)$.

The author would like to express her sincere gratitude to the referee for valuable suggestions.

\section{Preliminaries}

Let $C P^{m}$ denote the complex projective space of complex dimension $m$ with constant holomorphic sectional curvature 4 . We denote by $J$ the complex structure, and by $g$ the metric of $C P^{m}$.

Let $M$ be a real $n$-dimensional Riemannian manifold isometrically immersed in $C P^{m}$. We denote by the same $g$ the Riemannian metric on $M$ induced from $g$, and by $p$ the codimension of $M$, that is, $p=2 m-n$.

We denote by $T_{x}(M)$ and $T_{x}(M)^{\perp}$ the tangent space and the normal space of $M$ at $x$, respectively.

DEFINITION 1. A submanifold $M$ of a Kähler manifold $\tilde{M}$ with complex structure $J$ is called a $C R$ submanifold of $\tilde{M}$ if there exists a differentiable distribution $H: x \mapsto H_{x} \subset$ $T_{x}(M)$ on $M$ satisfying the following conditions:

(i) $H$ is holomorphic, i.e., $J H_{x}=H_{x}$ for each $x \in M$, and

(ii) the complementary orthogonal distribution $H^{\perp}: x \mapsto H_{x}^{\perp} \subset T_{x}(M)$ is antiinvariant, i.e. $J H_{x}^{\perp} \subset T_{x}(M)^{\perp}$ for each $x \in M$.

In the following, we put $\operatorname{dim} H_{x}=h$ and $\operatorname{dim} H_{x}^{\perp}=q$. If $q=0($ resp. $h=0)$, then the $C R$ submanifold $M$ is a complex submanifold (resp. totally real submanifold) of $\tilde{M}$. If $h>0$ and $q>0$, then a $C R$ submanifold $M$ is said to be proper.

We denote by $\tilde{\nabla}$ the operator of covariant differentiation in $C P^{m}$, and by $\nabla$ that in $M$ determined by the induced metric. Then the Gauss and Weingarten formulas are given respectively by

$$
\tilde{\nabla}_{X} Y=\nabla_{X} Y+B(X, Y), \quad \tilde{\nabla}_{X} V=-A_{V} X+D_{X} V
$$

for any vector fields $X$ and $Y$ tangent to $M$ and any vector field $V$ normal to $M$, where $D$ denotes the normal connection. We call both $A$ and $B$ the second fundamental form of $M$ and 
are related by $g(B(X, Y), V)=g\left(A_{V} X, Y\right)$. The second fundamental forms $A$ and $B$ are symmetric with respect to $X$ and $Y$.

The mean curvature vector of $M$ is defined to be the trace of the second fundamental form $B$, that is, $\operatorname{tr} B=\sum_{i} B\left(e_{i}, e_{i}\right),\left\{e_{i}\right\}$ being an orthonormal basis of $T_{x}(M)$. If the mean curvature vector vanishes identically, then $M$ is said to be minimal.

The covariant derivative $\left(\nabla_{X} A\right)_{V} Y$ of $A$ is defined by

$$
\left(\nabla_{X} A\right)_{V} Y=\nabla_{X}\left(A_{V} Y\right)-A_{D_{X} V} Y-A_{V} \nabla_{X} Y
$$

If $\left(\nabla_{X} A\right)_{V} Y=0$ for any vector fields $X$ and $Y$ tangent to $M$, then the second fundamental form of $M$ is said to be parallel in the direction of the normal vector $V$. If the second fundamental form is parallel in any direction, it is said to be parallel. A vector field $V$ normal to $M$ is said to be parallel if $D_{X} V=0$ for any vector field $X$ tangent to $M$.

For $x \in M$, the first normal space $N_{1}(x)$ is the orthogonal complement in $T_{x}(M)^{\perp}$ of the set $N_{0}(x)=\left\{V \in T_{x}(M)^{\perp}: A_{V}=0\right\}$. If $D_{X} V \in N_{1}(x)$ for any vector field $V$ with $V_{x} \in N_{1}(x)$ and any vector field $X$ of $M$ at $x$, then the first normal space $N_{1}(x)$ is said to be parallel with respect to the normal connection.

In the sequel, we assume that $M$ is a $C R$ submanifold of $C P^{m}$. The tangent space $T_{x}(M)$ of $M$ is decomposed as $T_{x}(M)=H_{x}+H_{x}^{\perp}$ at each point $x$ of $M$. Similarly, we see that $T_{x}(M)^{\perp}=J H_{x}^{\perp}+N_{x}$, where $N_{x}$ is the orthogonal complement of $J H_{x}^{\perp}$ in $T_{x}(M)^{\perp}$.

For any vector field $X$ tangent to $M$, we put

$$
J X=P X+F X,
$$

where $P X$ is the tangential part of $J X$ and $F X$ the normal part of $J X$. For any vector field $V$ normal to $M$, we put

$$
J V=t V+f V,
$$

where $t V$ is the tangential part of $J V$ and $f V$ the normal part of $J V$. Then we see that $F P=0, f F=0, t f=0$ and $P t=0$.

We define the covariant derivatives of $P, F, t$ and $f$ by $\left(\nabla_{X} P\right) Y=\nabla_{X}(P Y)-P \nabla_{X} Y$, $\left(\nabla_{X} F\right) Y=D_{X}(F Y)-F \nabla_{X} Y,\left(\nabla_{X} t\right) V=\nabla_{X}(t V)-t D_{X} V$ and $\left(\nabla_{X} f\right) V=D_{X}(f V)-$ $f D_{X} V$, respectively. We then have

$$
\begin{aligned}
& \left(\nabla_{X} P\right) Y=A_{F Y} X+t B(X, Y), \quad\left(\nabla_{X} F\right) Y=-B(X, P Y)+f B(X, Y), \\
& \left(\nabla_{X} t\right) V=-P A_{V} X+A_{f V} X, \quad\left(\nabla_{X} f\right) V=-F A_{V} X-B(X, t V) .
\end{aligned}
$$

For any vectors $X$ and $Y$ in $H_{x}^{\perp}=t T_{x}(M)^{\perp}$, we obtain $A_{F X} Y=A_{F Y} X$.

The Riemannian curvature tensor $\tilde{R}$ of a complex projective space $C P^{m}$ is given by

$$
\begin{aligned}
\tilde{R}(X, Y) Z= & g(Y, Z) X-g(X, Z) Y+g(J Y, Z) J X \\
& -g(J X, Z) J Y+2 g(X, J Y) J Z
\end{aligned}
$$


for any vector fields $X, Y$ and $Z$ of $C P^{m}$. Then the equation of Gauss and the equation of Codazzi are given respectively by

$$
\begin{aligned}
R(X, Y) Z= & g(Y, Z) X-g(X, Z) Y+g(P Y, Z) P X-g(P X, Z) P Y \\
& -2 g(P X, Y) P Z+A_{B(Y, Z)} X-A_{B(X, Z)} Y
\end{aligned}
$$

and

$$
\begin{aligned}
& g\left(\left(\nabla_{X} A\right)_{V} Y, Z\right)-g\left(\left(\nabla_{Y} A\right)_{V} X, Z\right) \\
& \quad=g(Y, P Z) g(X, t V)-g(X, P Z) g(Y, t V)-2 g(X, P Y) g(Z, t V)
\end{aligned}
$$

for any vector fields $X, Y$ and $Z$ tangent to $M$ and $V$ normal to $M$, where $R$ is the Riemannian curvature tensor field of $M$.

We denote by $S$ the Ricci tensor field of $M$. Then

$$
\begin{aligned}
S(X, Y)= & (n-1) g(X, Y)+3 g(P X, P Y) \\
& +\sum_{a} \operatorname{tr} A_{a} g\left(A_{a} X, Y\right)-\sum_{a} g\left(A_{a}^{2} X, Y\right),
\end{aligned}
$$

where $A_{a}$ is the second fundamental form in the direction of $v_{a},\left\{v_{1}, \ldots, v_{p}\right\}$ being an orthonormal basis of $T_{x}(M)^{\perp}$, and tr denotes the trace of an operator. If the Ricci tensor $S$ satisfies $S(X, Y)=\alpha g(X, Y)$ for some constant $\alpha$, then $M$ is called an Einstein manifold. When $M$ is a real hypersurface of $C P^{m}$ with a unit normal vector field $V$, if the Ricci tensor $S$ satisfies $S(X, Y)=\alpha g(X, Y)+\beta g(X, t V) g(Y, t V)$ for some constants $\alpha$ and $\beta$, then $M$ is said to be pseudo-Einstein.

We define the curvature tensor $R^{\perp}$ of the normal bundle $T(M)^{\perp}$ of $M$ by

$$
R^{\perp}(X, Y) V=D_{X} D_{Y} V-D_{Y} D_{X} V-D_{[X, Y]} V .
$$

Then we have the equation of Ricci:

$$
\begin{aligned}
& g\left(R^{\perp}(X, Y) V, U\right)+g\left(\left[A_{U}, A_{V}\right] X, Y\right) \\
& \quad=g(F Y, V) g(F X, U)-g(F X, V) g(F Y, U)+2 g(X, P Y) g(f V, U),
\end{aligned}
$$

where $[$,$] denotes the commutator and \left[A_{V}, A_{U}\right]=A_{V} A_{U}-A_{U} A_{V}$.

We need the following examples of $C R$ submanifolds in $C P^{m}$.

EXAMPLE 1 ([1]). An $n$-dimensional complete totally geodesic submanifold $M$ of $C P^{m}$ is either a complex projective space $C P^{n / 2}$ or a real projective space $R P^{n}$ of constant curvature 1. A real projective space $R P^{n}$ is a totally real submanifold of $C P^{m}$.

EXAMPLE 2. Let $z^{0}, z^{1}, \ldots, z^{m}$ be homogeneous coordinates of $C P^{m}$. The complex quadric $Q^{m-1}$ is a complex hypersurface of $C P^{m}$ defined by the equation

$$
\left(z^{0}\right)^{2}+\left(z^{1}\right)^{2}+\cdots+\left(z^{m}\right)^{2}=0 .
$$


Then $Q^{m-1}$ is a Kähler manifold. Moreover, $Q^{m-1}$ is an Einstein manifold with Ricci curvature $2(m-1)$ (see [13]).

EXAMPLE 3. For an integer $k$ and for $0<r<\pi / 2$, we define $M(k, r)$ in $S^{2 m+1}$ by

$$
\sum_{j=0}^{k}\left|z_{j}\right|^{2}=\cos ^{2} r, \quad \sum_{j=k+1}^{m}\left|z_{j}\right|^{2}=\sin ^{2} r .
$$

$M(k, r)$ is a standard product $S^{2 k+1}(\cos r) \times S^{2 l+1}(\sin r), l=m-k-1$. We consider the Hopf fibration $\pi: S^{2 m+1} \longrightarrow C P^{m}$, where $S^{2 m+1}$ denotes the unit sphere. Then $M^{c}(k, r)=$ $\pi(M(k, r))$ is a real hypersurface in $C P^{m}$. For an integer $1 \leq k \leq m-2$, we see that $M^{c}(k, r)$ is the tube of radius $r$ over $C P^{k}$ (see [3]).

When $r$ satisfies $\cos r=\sqrt{(2 k+1) /(2 m)}$ and $\sin r=\sqrt{(2 l+1) /(2 m)}, M^{c}(k, r)$ is a minimal real hypersurface of $C P^{m}$. Moreover, we see that $M^{c}(k, r)$ is a pseudo-Einstein real minimal hypersurface of $C P^{m}$ if and only if $k=l=(m-1) / 2$ and $r=\pi / 4$. Then the Ricci tensor $S$ satisfies $S(X, Y)=(2 m-2) g(X, Y)+2 g(P X, P Y)$.

\section{Integral formula}

In this section, for later use, we compute the Laplacian for the square of the length of the second fundamental form $A$ of an $n$-dimensional minimal submanifold $M$ immersed in a complex projective space $C P^{m}$. In the following, we put $\nabla_{i}=\nabla_{e_{i}}$ and $D_{i}=D_{e_{i}}$, where $\left\{e_{i}\right\}$ being an orthonormal basis of $M$, to simplify the notation. We use the following (see Simons [12])

Lemma 1. Let $M$ be a submanifold of a locally symmetric Riemannian manifold $\bar{M}$. If the mean curvature vector field of $M$ is parallel, then

$$
\begin{aligned}
g( & \left.\left(\nabla^{2} B\right)(X, Y), V\right)=\sum_{i} g\left(\left(\nabla_{i} \nabla_{i} B\right)(X, Y), V\right) \\
= & \sum_{i}\left(2 g\left(\bar{R}\left(e_{i}, Y\right) B\left(X, e_{i}\right), V\right)+2 g\left(\bar{R}\left(e_{i}, X\right) B\left(Y, e_{i}\right), V\right)\right. \\
& \quad-g\left(A_{V} X, \bar{R}\left(e_{i}, Y\right) e_{i}\right)-g\left(A_{V} Y, \bar{R}\left(e_{i}, X\right) e_{i}\right)+g\left(\bar{R}\left(e_{i}, B(X, Y)\right) e_{i}, V\right) \\
& \left.\quad+g\left(\bar{R}\left(B\left(e_{i}, e_{i}\right), X\right) Y, V\right)-2 g\left(A_{V} e_{i}, \bar{R}\left(e_{i}, X\right) Y\right)\right) \\
+ & \sum_{a}\left(\operatorname{tr} A_{a} g\left(A_{V} A_{a} X, Y\right)-\operatorname{tr} A_{a} A_{V} g\left(A_{a} X, Y\right)+2 g\left(A_{a} A_{V} A_{a} X, Y\right)\right. \\
& \left.\quad-g\left(A_{a}^{2} A_{V} X, Y\right)-g\left(A_{V} A_{a}^{2} X, Y\right)\right)
\end{aligned}
$$

for any vectors $X, Y$ tangent to $M$ and any vector $V$ normal to $M$. 
We compute the equation of Lemma 1 for an $n$-dimensional minimal submanifold $M$ in $C P^{m}$. We notice that $C P^{m}$ is locally symmetric. Using the expression of the curvature tensor $\tilde{R}$ of $C P^{m}$, we have the equation of Lemma 1 in the following:

$$
\begin{aligned}
g\left(\left(\nabla^{2} B\right)(X, Y), V\right) & \\
= & \sum_{i} g\left(\left(\nabla_{i} \nabla_{i} B\right)(X, Y), V\right) \\
= & -2 g\left(A_{F Y} X, t V\right)-2 g\left(A_{F X} Y, t V\right) \\
& +2 \sum_{i} g(Y, t V) g\left(A_{F e_{i}} e_{i}, X\right)+2 \sum_{i} g(X, t V) g\left(A_{F e_{i}} e_{i}, Y\right) \\
& -4 g\left(A_{f V} X, P Y\right)-4 g\left(A_{f V} Y, P X\right) \\
& +n g\left(A_{V} X, Y\right)-3 g\left(A_{V} X, P^{2} Y\right)-3 g\left(A_{V} Y, P^{2} X\right) \\
& +3 g\left(A_{F t V} X, Y\right)-6 g\left(A_{V} P X, P Y\right) \\
& +\sum_{a}\left(-\operatorname{tr} A_{a} A_{V} g\left(A_{a} X, Y\right)+2 g\left(A_{a} A_{V} A_{a} X, Y\right)\right. \\
& \left.\quad-g\left(A_{a}^{2} A_{V} X, Y\right)-g\left(A_{V} A_{a}^{2} X, Y\right)\right) .
\end{aligned}
$$

We have $g\left(\left(\nabla^{2} B\right)(X, Y), V\right)=g\left(\left(\nabla^{2} A\right)_{V} X, Y\right)$. Hence

$$
\begin{aligned}
g\left(\nabla^{2} A, A\right) & \\
= & n \sum_{a} \operatorname{tr} A_{a}^{2}-3 \sum_{a, b} \operatorname{tr} A_{a} A_{b} g\left(t v_{a}, t v_{b}\right)-6 \sum_{a} \operatorname{tr} P^{2} A_{a}^{2}+6 \sum_{a}\left(\operatorname{tr} A_{a} P\right)^{2} \\
& +4 \sum_{a, b}\left(g\left(A_{a} t v_{b}, A_{b} t v_{a}\right)-g\left(A_{a} t v_{a}, A_{b} t v_{b}\right)\right)-8 \sum_{a} \operatorname{tr} A_{a} A_{f a} P \\
& +\sum_{a, b}\left(-\left(\operatorname{tr} A_{a} A_{b}\right)^{2}+2 \operatorname{tr}\left(A_{a} A_{b}\right)^{2}-2 \operatorname{tr} A_{a}^{2} A_{b}^{2}\right),
\end{aligned}
$$

where we put $A_{f a}=A_{f v_{a}}$. Moreover we obtain

$$
\begin{aligned}
& \begin{aligned}
& \sum_{a, b} \operatorname{tr} A_{a} A_{b} g\left(t v_{a}, t v_{b}\right)=\sum_{a} \operatorname{tr} A_{a}^{2}-\sum_{a, b} \operatorname{tr} A_{a} A_{b} g\left(f v_{a}, f v_{b}\right) \\
&=\sum_{a} \operatorname{tr} A_{a}^{2}-\sum_{a, b, c} \operatorname{tr} A_{a} A_{b} g\left(f v_{a}, v_{c}\right) g\left(f v_{b}, v_{c}\right) \\
&=\sum_{a} \operatorname{tr} A_{a}^{2}-\sum_{a, b, c, i} g\left(A_{a} e_{i}, A_{b} e_{i}\right) g\left(v_{a}, f v_{c}\right) g\left(v_{b}, f v_{c}\right) \\
&=\sum_{a} \operatorname{tr} A_{a}^{2}-\sum_{a} \operatorname{tr} A_{f a}^{2}, \\
& 2 \sum_{a, b}\left(\operatorname{tr} A_{a}^{2} A_{b}^{2}-\operatorname{tr}\left(A_{a} A_{b}\right)^{2}\right)=\sum_{a, b}\left|\left[A_{a}, A_{b}\right]\right|^{2}
\end{aligned}
\end{aligned}
$$




$$
2 \sum_{a}\left(\operatorname{tr}\left(A_{a} P\right)^{2}-\operatorname{tr} A_{a}^{2} P^{2}\right)=\sum_{a}\left|\left[P, A_{a}\right]\right|^{2},
$$

where $|\cdot|$ denotes the length of the tensor. Therefore we have the following theorem.

THEOREM 1. Let $M$ be an n-dimensional minimal submanifold of a complex projective space $C P^{m}$. Then we have

$$
\begin{aligned}
g\left(\nabla^{2} A, A\right) & \\
= & (n-3) \sum_{a} \operatorname{tr} A_{a}^{2}+3 \sum_{a} \operatorname{tr} A_{f a}^{2} \\
& +4 \sum_{a, b}\left(g\left(A_{a} t v_{b}, A_{b} t v_{a}\right)-g\left(A_{a} t v_{a}, A_{b} t v_{b}\right)\right)-8 \sum_{a} \operatorname{tr} A_{a} A_{f a} P \\
& +3 \sum_{a}\left|\left[P, A_{a}\right]\right|^{2}-\sum_{a, b}\left|\left[A_{a}, A_{b}\right]\right|^{2}-\sum_{a, b}\left(\operatorname{tr} A_{a} A_{b}\right)^{2} .
\end{aligned}
$$

\section{Reduction of the codimension}

In this section we prove the following reduction theorem for the codimension.

THEOREM 2. Let $M$ be a compact $n$-dimensional minimal proper $C R$ submanifold of a complex projective space $C P^{m}$. If the Ricci tensor $S$ of $M$ satisfies $S(X, X) \geq$ $(n-1) g(X, X)$ for any vector field $X$ tangent to $M$, then $M$ is a real hypersurface of some $C P^{(n+1) / 2}$ in $C P^{m}$.

First of all, we prove

Lemma 2. Let $M$ be a compact n-dimensional minimal $C R$ submanifold of $C P^{m}$ which is not a complex submanifold of $C P^{m}$. If the Ricci tensor $S$ of $M$ satisfies $S(X, X) \geq$ $(n-1) g(X, X)$, then $M$ is a real projective space $R P^{n}$ or $q=1$, that is, $\operatorname{dim} H_{x}^{\perp}=1$.

Proof. Since $M$ is minimal, by the assumption, we have

$$
S(X, X)-(n-1) g(X, X)=3 g(P X, P X)-\sum_{a} g\left(A_{a}^{2} X, X\right) \geq 0 .
$$

If $P=0$, then $M$ is a totally real submanifold of $C P^{m}$. Moreover the above inequality implies that $A_{a}=0$ for all $a$. So $M$ is totally geodesic in $C P^{m}$, and hence $M$ is a real projective space $R P^{n}$ by a theorem of Abe [1].

We next suppose that $P \neq 0$. For any normal vector fields $U$ and $V$, we have $A_{U} t V=0$. Using this,

$$
0=\left(\nabla_{X} A\right)_{U} t V-A_{U} P A_{V} X+A_{U} A_{f V} X
$$

from which

$$
g\left(\left(\nabla_{X} A\right)_{U} Y, t V\right)=g\left(\left(\nabla_{X} A\right)_{U} t V, Y\right)
$$




$$
=g\left(A_{U} P A_{V} X, Y\right)-g\left(A_{U} A_{f V} X, Y\right) .
$$

So the equation of Codazzi implies

$$
\begin{aligned}
-2 g(X, P Y) g(t U, t V)= & g\left(A_{U} P A_{V} X, Y\right)+g\left(A_{V} P A_{U} X, Y\right) \\
& -g\left(A_{U} A_{f V} X, Y\right)+g\left(A_{f V} A_{U} X, Y\right) .
\end{aligned}
$$

Since $\sum_{a} g\left(t v_{a}, t v_{a}\right)=q$, it follows that

$$
\begin{aligned}
& 2 \sum_{a} g\left(A_{a} P A_{a} X, P X\right)-\sum_{a} g\left(\left(A_{a} A_{f a}-A_{f a} A_{a}\right) X, P X\right) \\
& \quad=2 q g(P X, P X) .
\end{aligned}
$$

On the other hand, we have

$$
S(P X, P X)=(n+2) g(P X, P X)-\sum_{a} g\left(A_{a} P X, A_{a} P X\right) .
$$

These equations imply

$$
\begin{aligned}
\sum_{a} g\left(A_{a} P X, A_{a} P X\right)= & \sum_{a} g\left(A_{a} P A_{a} X, P X\right)-\frac{1}{2} \sum_{a} g\left(\left(A_{a} A_{f a}-A_{f a} A_{a}\right) X, P X\right) \\
& +(n+2-q) g(P X, P X)-S(P X, P X) .
\end{aligned}
$$

Thus we have, for any orthonormal basis $\left\{e_{i}\right\}$ of $T_{x}(M)$,

$$
\begin{aligned}
& \frac{1}{2} \sum_{a}\left|\left[P, A_{a}\right]\right|^{2} \\
& \quad=(n+2-q) h-\sum_{i} S\left(P e_{i}, P e_{i}\right)+\frac{1}{2} \sum_{a} \operatorname{tr} P\left(A_{a} A_{f a}-A_{f a} A_{a}\right) \\
& \quad=-h q+\sum_{a} \operatorname{tr} A_{a}^{2}+\sum_{a} \operatorname{tr} P A_{a} A_{f a} .
\end{aligned}
$$

By (2), we obtain $\sum_{a} \operatorname{tr} A_{a}^{2} \leq 3 h$. From these,

$$
\frac{1}{2} \sum_{a}\left|\left[P, A_{a}\right]\right|^{2} \leq h(3-q)+\sum_{a} \operatorname{tr} P A_{a} A_{f a} .
$$

We take a basis $\left\{v_{1}, \ldots, v_{p}\right\}$ of $T_{x}(M)^{\perp}$ such that $\left\{v_{1}, \ldots, v_{q}\right\}$ is an orthonormal basis of $F T_{x}(M)$ and $\left\{v_{q+1}, \ldots, v_{p}\right\}$ is that of $N_{x}$. We denote by the same $\left\{v_{1}, \ldots, v_{p}\right\}$ an orthonormal normal vector fields in a neighborhood of $x$. By (3), we have $\sum_{\lambda=q+1}^{p} \operatorname{tr} P A_{\lambda} A_{f \lambda}=$ $\sum_{\lambda=q+1}^{p} \operatorname{tr} A_{\lambda} P A_{\lambda} P$. From these and

$$
\frac{1}{2} \sum_{a=1}^{p}\left|\left[P, A_{a}\right]\right|^{2}=\frac{1}{2} \sum_{y=1}^{q}\left|\left[P, A_{y}\right]\right|^{2}+\sum_{\lambda=q+1}^{p} \operatorname{tr} A_{\lambda} P A_{\lambda} P-\sum_{\lambda=q+1}^{p} \operatorname{tr} P^{2} A_{\lambda}^{2},
$$


we obtain

$$
0 \leq \frac{1}{2} \sum_{y=1}^{q}\left|\left[P, A_{y}\right]\right|^{2}+\sum_{i=1}^{n} \sum_{\lambda=q+1}^{p} g\left(A_{\lambda} P e_{i}, A_{\lambda} P e_{i}\right) \leq h(3-q) .
$$

Thus we see that $q \leq 3$. Suppose $q=3$. Then, $P A_{y}=A_{y} P$ for $y=1,2,3$ and $A_{\lambda} P=0$ for $\lambda=4, \ldots, p$. Hence we have $A_{f V} P X=0$ for any normal vector $V$ and tangent vector $X$. Then, it follows from (3) that

$$
2 g(P X, P Y) g(t V, t U)=g\left(A_{U} A_{V} P X, P Y\right)+g\left(A_{V} A_{U} P X, P Y\right)
$$

for any tangent vectors $X, Y$ and normal vectors $U, V \in F T_{X}(M)$. So we see that if $g(U, V)=0$, then $A_{U} A_{V}+A_{V} A_{U}=0$. Moreover, $A_{y}^{2} X=X$ and $g\left(A_{y} X, A_{z} X\right)=$ $g(X, X) g\left(t v_{y}, t v_{z}\right)$ for any $X \in H_{x}$ and $y, z=1,2,3$. We denote by $H_{1}$ and $H_{2}$ the eigenspaces of $A_{1}$ corresponding to 1 and -1 , respectively. If $X \in H_{1}$, then $A_{1} A_{2} X=$ $-A_{2} A_{1} X=-A_{2} X$ and $A_{1} A_{3} X=-A_{3} A_{1} X=-A_{3} X$. So we have $A_{2} X \in H_{2}$ and $A_{3} X \in H_{2}$. Similarly, if $X \in H_{2}$, then $A_{2} X \in H_{1}$ and $A_{3} X \in H_{1}$. We can take an orthonormal basis $\left\{e_{i}\right\}$ of $H_{1}$ which satisfies $A_{1} e_{i}=e_{i}, i=1, \ldots, s$, where $s=\operatorname{dim} H_{1}=\operatorname{dim} H_{2}$ since $M$ is minimal. Then $A_{1}$ can be diagonalized with respect to the orthonormal basis $\left\{e_{1}, \ldots, e_{s}, A_{2} e_{1}, \ldots, A_{2} e_{s}, e_{2 s+1}, \ldots, e_{n}\right\}$. Then, for $e_{i}, e_{j} \in H_{1}$,

$$
g\left(A_{2} e_{i}, e_{j}\right)=0, \quad g\left(A_{2}^{2} e_{i}, A_{2} e_{j}\right)=0, \quad g\left(A_{2} e_{i}, A_{2} e_{j}\right)=\delta_{i j} .
$$

So $A_{2}$ can be represented by a matrix of the form

$$
A_{2}=\left(\begin{array}{c|c|c}
0 & I_{s} & 0 \\
\hline I_{s} & 0 & 0 \\
\hline 0 & 0 & 0
\end{array}\right)
$$

where $I_{s}$ denotes the identity matrix of degree $s$. Similarly,

$$
A_{3}=\left(\begin{array}{l|l|l}
0 & * & 0 \\
\hline * & 0 & 0 \\
\hline 0 & 0 & 0
\end{array}\right)
$$

Thus we obtain $A_{2} A_{3}=A_{3} A_{2}$. Since $A_{2} A_{3}+A_{3} A_{2}=0$, we have $A_{2} A_{3}=A_{3} A_{2}=0$. Hence $A_{2}=A_{3}^{2} A_{2}=0$. This is a contradiction .

Suppose $q=2$. We have $A_{f y}=0$ for $y=1,2$. Then

$$
\begin{aligned}
& \sum_{y, i, j} g\left(\nabla_{j} t v_{y}, e_{i}\right) g\left(e_{j}, \nabla_{i} t v_{y}\right) \\
& =\sum_{y, i, j} g\left(-P A_{y} e_{j}+t D_{j} v_{y}, e_{i}\right) g\left(-P A_{y} e_{i}+t D_{i} v_{y}, e_{j}\right) \\
& =-\sum_{y, j} g\left(P A_{y} e_{j}, A_{y} P e_{j}\right)+\sum_{y, i, j} g\left(t D_{j} v_{y}, e_{i}\right) g\left(t D_{i} v_{y}, e_{j}\right)
\end{aligned}
$$




$$
\begin{aligned}
& =\sum_{y} \operatorname{tr}\left(P A_{y}\right)^{2}+\sum_{y, z, w} g\left(D_{t w} v_{y}, v_{z}\right) g\left(D_{t z} v_{y}, v_{w}\right) \\
& =\sum_{y} \operatorname{tr}\left(P A_{y}\right)^{2}+\sum_{y, z} g\left(D_{t z} v_{y}, v_{z}\right)^{2}
\end{aligned}
$$

where $y, z, w=1,2$ and $D_{t y}=D_{t v_{y}}$. On the other hand, we have

$$
\begin{aligned}
\sum_{y}\left(\operatorname{div} t v_{y}\right)^{2} & =\sum_{y, i, j} g\left(\nabla_{i} t v_{y}, e_{i}\right) g\left(\nabla_{j} t v_{y}, e_{j}\right) \\
& =\sum_{y, i, j} g\left(-P A_{y} e_{i}+t D_{i} v_{y}, e_{i}\right) g\left(-P A_{y} e_{j}+t D_{j} v_{y}, e_{j}\right) \\
& =\sum_{y, i, j} g\left(t D_{i} v_{y}, e_{i}\right) g\left(t D_{j} v_{y}, e_{j}\right) \\
& =\sum_{y, z} g\left(D_{t z} v_{y}, v_{z}\right)^{2}
\end{aligned}
$$

Since $S$ satisfies

$$
\begin{aligned}
& \operatorname{div}\left(\nabla_{X} X\right)-\operatorname{div}((\operatorname{div} X) X) \\
& \quad=S(X, X)+\sum_{i, j} g\left(\nabla_{j} X, e_{i}\right) g\left(e_{j}, \nabla_{i} X\right)-(\operatorname{div} X)^{2}
\end{aligned}
$$

for any tangent vector field $X$ (cf. [15; p. 44]), it follows that

$$
\begin{aligned}
& \sum_{y}\left(\operatorname{div}\left(\nabla_{t y} t v_{y}\right)-\operatorname{div}\left(\left(\operatorname{div} t v_{y}\right) t v_{y}\right)\right) \\
& \quad=\sum_{y} S\left(t v_{y}, t v_{y}\right)+\sum_{y} \operatorname{tr}\left(P A_{y}\right)^{2} \\
& \quad=2(n-1)+\frac{1}{2} \sum_{y}\left|\left[P, A_{y}\right]\right|^{2}+\sum_{y} \operatorname{tr}\left(P^{2} A_{y}^{2}\right) \\
& \quad=2(n-1)-2 h+\sum_{y} \operatorname{tr} A_{y}^{2}+\sum_{y} \operatorname{tr} P A_{y} A_{f y}+\sum_{y} \operatorname{tr}\left(P^{2} A_{y}^{2}\right) \\
& \quad \geq 2 .
\end{aligned}
$$

Here we used (4) and $f v_{y}=0$. However, since $M$ is compact, this is a contradiction. So we have $q=1$.

If $M$ is proper, then $h>0$ and $q>0$. Then Lemma 2 reduces to

LEMMA 3. Let $M$ be a compact $n$-dimensional minimal proper $C R$ submanifold of $C P^{m}$. If the Ricci tensor $S$ of $M$ satisfies $S(X, X) \geq(n-1) g(X, X)$, then $q=1$, that is, $\operatorname{dim} H_{x}^{\perp}=1$. 
In the following, we shall prove that the first normal space of $M$ is just $F H_{x}^{\perp}$ and is of dimension 1 under the condition of Lemma 3. To prove this, we prepare some lemmas.

LEMMA 4. Let $M$ be a compact $n$-dimensional minimal proper $C R$ submanifold of $C P^{m}$. If the Ricci tensor $S$ of $M$ satisfies $S(X, X) \geq(n-1) g(X, X)$, then the following hold:

(a) $\nabla f=0$.

(b) For any $X$ tangent to $M$ and any $V \in F H^{\perp}$, we have $D_{X} V \in F H^{\perp}$.

(c) For any $X$ tangent to $M$ and any $U \in N$, we have $D_{X} U \in N$.

PROOF. By the proof of Lemma 2, if the Ricci tensor $S$ of a minimal $C R$ submanifold $M$ satisfies $S(X, X) \geq(n-1) g(X, X)$ for any tangent vector field $X$, then $A_{U} t V=0$ for any $U$ and $V$ normal to $M$. Thus we have

$$
\begin{aligned}
g\left(\left(\nabla_{X} f\right) V, U\right) & =-g\left(F A_{V} X, U\right)-g(B(X, t V), U) \\
& =g\left(X, A_{V} t U\right)-g\left(A_{U} t V, X\right) \\
& =0
\end{aligned}
$$

for any $X$ tangent to $M$ and any $U$ and $V$ normal to $M$. This means that $f$ is parallel.

Since $M$ is proper, by Lemma 3, we have $\operatorname{dim} H_{x}^{\perp}=1$. Let $V$ be a vector field in $F H^{\perp}$. Then we see $g\left(D_{X} V, f U\right)=-g\left(V,\left(\nabla_{X} f\right) U\right)=0$ for any vector field $U \in N$. This proves (b).

Next we prove (c). For any vector field $U$ in $N$, there exists $U^{\prime}$ in $N$ such that $U=f U^{\prime}$. Therefore we have

$$
D_{X} U=D_{X}\left(f U^{\prime}\right)=f D_{X} U^{\prime}
$$

This shows $D_{X} U \in N$.

LEMMA 5. Let $M$ be a compact $n$-dimensional minimal proper $C R$ submanifold of $C P^{m}$. If the Ricci tensor $S$ of $M$ satisfies $S(X, X) \geq(n-1) g(X, X)$, then the second fundamental form A satisfies the following:

(a) $A_{v} P A_{v}=P$, where $v$ is a unit vector field in $F H^{\perp}$.

(b) $\left|\left[P, A_{v}\right]\right|^{2}=2 \operatorname{tr} A_{v}^{2}-2(n-1)$, where $v$ is a unit vector field in $F H^{\perp}$.

(c) $A_{V} A_{U}=A_{U} A_{V}$ for any $V \in F H^{\perp}$ and $U \in N$.

(d) $P A_{U}=A_{f U}$ and $P A_{U}+A_{U} P=0$ for any $U \in N$.

PROOF. By Lemma 3, we have $\operatorname{dim} H_{x}^{\perp}=1$. Let $\left\{v_{1}, \ldots, v_{p}\right\}$ be an orthonormal basis of $T_{x}(M)^{\perp}$ such that $v_{1}=v \in F H_{x}^{\perp}$ and $v_{2}, \ldots, v_{p} \in N_{x}$.

By (3) and $f v=0$, we obtain

$$
2 g\left(A_{v} P A_{v} X, Y\right)=-2 g(X, P Y) g(t v, t v)
$$

for any $X$ and $Y$ tangent to $M$. Thus we have (a). Using this, we can prove (b) by a straightforward computation.

Next we prove (c). From the equation of Ricci and Lemma 4 (b), we see

$$
g\left(\left[A_{U}, A_{V}\right] X, Y\right)
$$




$$
\begin{aligned}
& =g(Y, t V) g(X, t U)-g(X, t V) g(Y, t U)-2 g(X, P Y) g(V, f U) \\
& =0
\end{aligned}
$$

for any $X$ and $Y$ tangent to $M$ and $V \in F H^{\perp}, U \in N$. This shows (c).

From the Weingarten formula and Lemma 4 (a), we have

$$
\tilde{\nabla}_{X} J U=\tilde{\nabla}_{X} f U=-A_{f U} X+D_{X} f U=-A_{f U} X+f D_{X} U .
$$

On the other hand, it follows from $\tilde{\nabla} J=0$ and Lemma 4 (c) that

$$
\tilde{\nabla}_{X} J U=J \tilde{\nabla}_{X} U=-P A_{U} X-F A_{U} X+f D_{X} U,
$$

from which $P A_{U}=A_{f U}$. Since $A_{f U}$ is symmetric and $P$ is skew-symmetric, we obtain $P A_{U}+A_{U} P=0$. This proves (d).

Using Theorem 1 and Lemma 5, we next compute the Laplacian for the square of the length of the second fundamental form of the minimal submanifold in $C P^{m}$ whose Ricci tensor satisfies $S(X, X) \geq(n-1) g(X, X)$ for any tangent vector field $X$.

LEMMA 6. Let $M$ be a compact n-dimensional minimal proper $C R$ submanifold of $C P^{m}$. If the Ricci tensor $S$ of $M$ satisfies $S(X, X) \geq(n-1) g(X, X)$, then

$$
\begin{aligned}
g\left(\nabla^{2} A, A\right)= & (n+3) \operatorname{tr} A_{v}^{2}+(n+4) \sum_{a} \operatorname{tr} A_{f a}^{2}-6(n-1) \\
& -\sum_{a, b}\left|\left[A_{a}, A_{b}\right]\right|^{2}-\sum_{a, b}\left(\operatorname{tr} A_{a} A_{b}\right)^{2} .
\end{aligned}
$$

ProOF. From Lemma 5, we have $\sum_{a} \operatorname{tr} A_{a} A_{f a} P=\sum_{a} \operatorname{tr} A_{f a}^{2}$. Next we compute $\sum_{a}\left|\left[P, A_{a}\right]\right|^{2}$. Using Lemma 5,

$$
\begin{aligned}
\sum_{a}\left|\left[P, A_{a}\right]\right|^{2} & =\left|\left[P, A_{v}\right]\right|^{2}+\sum_{a \geq 2}\left|\left[P, A_{a}\right]\right|^{2} \\
& =-2(n-1)+2 \operatorname{tr} A_{v}^{2}+4 \sum_{a} \operatorname{tr} A_{f a}^{2} .
\end{aligned}
$$

From these equations and Theorem 1, we have our result.

LEMMA 7. Let $M$ be a compact $n$-dimensional minimal proper $C R$ submanifold of $C P^{m}$. If the Ricci tensor $S$ of $M$ satisfies $S(X, X) \geq(n-1) g(X, X)$, then

$$
\begin{aligned}
& \sum_{j} g\left(\left(\nabla^{2} A\right)_{v} e_{j}, A_{v} e_{j}\right)=(n+3) \operatorname{tr} A_{v}^{2}-6(n-1)-\left(\operatorname{tr} A_{v}^{2}\right)^{2}, \\
& \sum_{a \geq 2, j} g\left(\left(\nabla^{2} A\right)_{a} e_{j}, A_{a} e_{j}\right)=(n+4) \sum_{a} \operatorname{tr} A_{f a}^{2}-\sum_{a, b}\left|\left[A_{a}, A_{b}\right]\right|^{2}-\sum_{a, b \geq 2}\left(\operatorname{tr} A_{a} A_{b}\right)^{2} .
\end{aligned}
$$


PROOF. By Lemma 5 (c) and (d), for any $v_{a} \in N$,

$$
\begin{aligned}
\operatorname{tr} A_{a} A_{v} & =-\operatorname{tr} A_{f^{2} a} A_{v}=-\operatorname{tr} P A_{f a} A_{v}=-\operatorname{tr} A_{f a} A_{v} P=-\operatorname{tr} A_{v} A_{f a} P \\
& =\operatorname{tr} A_{v} P A_{f a}=\operatorname{tr} A_{v} A_{f^{2} a}=-\operatorname{tr} A_{v} A_{a}=-\operatorname{tr} A_{a} A_{v} .
\end{aligned}
$$

Hence we have $\operatorname{tr} A_{a} A_{v}=0$. Thus, using (1) and Lemma 5, we have

$$
\begin{aligned}
\sum_{j} g & \left(\left(\nabla^{2} A\right)_{v} e_{j}, A_{v} e_{j}\right) \\
= & \sum_{j} g\left(\left(\nabla^{2} B\right)\left(e_{j}, A_{v} e_{j}\right), v\right) \\
= & n g \sum_{j} g\left(A_{v} e_{j}, A_{v} e_{j}\right)-3 \sum_{j} g\left(A_{v} e_{j}, P^{2} A_{v} e_{j}\right) \\
& -3 \sum_{j} g\left(A_{v}^{2} e_{j}, P^{2} e_{j}\right)-3 \sum_{j} g\left(A_{v} e_{j}, A_{v} e_{j}\right)-6 \sum_{j} g\left(A_{v} P e_{j}, P A_{v} e_{j}\right) \\
& +\sum_{a, j}\left(-\operatorname{tr} A_{a} A_{v} g\left(A_{a} e_{j}, A_{v} e_{j}\right)+2 g\left(A_{a} A_{v} A_{a} e_{j}, A_{v} e_{j}\right)\right. \\
& \left.-g\left(A_{a}^{2} A_{v} e_{j}, A_{v} e_{j}\right)-g\left(A_{v} A_{a}^{2} e_{j}, A_{v} e_{j}\right)\right) \\
= & (n-3) \operatorname{tr} A_{v}^{2}+3\left|\left[P, A_{v}\right]\right|^{2}-\sum_{a}\left(\operatorname{tr} A_{a} A_{v}\right)^{2}+\sum_{a}\left|\left[A_{a}, A_{v}\right]\right|^{2} \\
= & (n+3) \operatorname{tr} A_{v}^{2}-6(n-1)-\left(\operatorname{tr} A_{v}^{2}\right)^{2} .
\end{aligned}
$$

From this equation and Lemma 6, we obtain

$$
\begin{aligned}
& \sum_{a \geq 2, j} g\left(\left(\nabla^{2} A\right)_{a} e_{j}, A_{a} e_{j}\right) \\
& \quad=g\left(\nabla^{2} A, A\right)-\sum_{j} g\left(\left(\nabla^{2} A\right)_{v} e_{j}, A_{v} e_{j}\right) \\
& \quad=(n+4) \sum_{a} \operatorname{tr} A_{f a}^{2}-\sum_{a, b}\left|\left[A_{a}, A_{b}\right]\right|^{2}-\sum_{a, b}\left(\operatorname{tr} A_{a} A_{b}\right)^{2}+\left(\operatorname{tr} A_{v}^{2}\right)^{2} \\
& \quad=(n+4) \sum_{a} \operatorname{tr} A_{f a}^{2}-\sum_{a, b}\left|\left[A_{a}, A_{b}\right]\right|^{2}-\sum_{a, b \geq 2}\left(\operatorname{tr} A_{a} A_{b}\right)^{2} .
\end{aligned}
$$

Hence we have our equation.

Next we give inequalities for $\sum_{a, b}\left|\left[A_{a}, A_{b}\right]\right|^{2}$ and $\sum_{a, b \geq 2}\left(\operatorname{tr} A_{a} A_{b}\right)^{2}$ in the equation in Lemma 7.

LEMMA 8. Let $M$ be a compact $n$-dimensional minimal proper $C R$ submanifold of 
$C P^{m}$. If the Ricci tensor $S$ of $M$ satisfies $S(X, X) \geq(n-1) g(X, X)$, then

$$
\begin{aligned}
& \sum_{a, b}\left|\left[A_{a}, A_{b}\right]\right|^{2} \leq 4 \sum_{a} \operatorname{tr} A_{f a}^{2}, \\
& \sum_{a, b \geq 2}\left(\operatorname{tr} A_{a} A_{b}\right)^{2} \leq \frac{1}{2}\left(\sum_{a} \operatorname{tr} A_{f a}^{2}\right)^{2} .
\end{aligned}
$$

Proof. From (2), we have $3 g(P X, P X) \geq \sum_{a} g\left(A_{a} X, A_{a} X\right)$ for any $X$ tangent to $M$. On the other hand, by Lemma 5 ,

$$
\begin{aligned}
& \sum_{i, a} g\left(A_{v}^{2} A_{f a} e_{i}, A_{f a} e_{i}\right) \\
& =\sum_{i, a} g\left(A_{v} A_{f a} A_{v} e_{i}, A_{f a} e_{i}\right)=\sum_{i, a} g\left(A_{v} P A_{a} A_{v} e_{i}, A_{f a} e_{i}\right) \\
& =\sum_{i, a} g\left(A_{v} P A_{v} A_{a} e_{i}, A_{f a} e_{i}\right)=\sum_{i, a \geq 2} g\left(P A_{a} e_{i}, P A_{a} e_{i}\right) .
\end{aligned}
$$

Using these and Lemma 5, we obtain

$$
\begin{aligned}
3 \sum_{a} \operatorname{tr} A_{f a}^{2} & =3 \sum_{i, a} g\left(P A_{f a} e_{i}, P A_{f a} e_{i}\right) \\
& \geq \sum_{i, a, b} g\left(A_{b} A_{f a} e_{i}, A_{b} A_{f a} e_{i}\right) \\
& =\sum_{i, a} g\left(A_{v} A_{f a} e_{i}, A_{v} A_{f a} e_{i}\right)+\sum_{i, a, b} g\left(A_{f a}^{2} A_{f b}^{2} e_{i}, e_{i}\right) \\
& =\sum_{i, a \geq 2} g\left(P A_{a} e_{i}, P A_{a} e_{i}\right)+\frac{1}{2} \sum_{a, b}\left|\left[A_{a}, A_{b}\right]\right|^{2} \\
& =\sum_{a} \operatorname{tr} A_{f a}^{2}+\frac{1}{2} \sum_{a, b}\left|\left[A_{a}, A_{b}\right]\right|^{2},
\end{aligned}
$$

from which $4 \sum_{a} \operatorname{tr} A_{f a}^{2} \geq \sum_{a, b}\left|\left[A_{a}, A_{b}\right]\right|^{2}$. Hence our first inequality holds. In the next place, we take a basis $\left\{v, v_{2}, \ldots, v_{p^{\prime}}, v_{p^{\prime}+1}=f v_{2}, \ldots, v_{p}=f v_{p^{\prime}}\right\}\left(p=2 p^{\prime}+1\right)$ of $T_{x}(M)^{\perp}$ such that $\sum_{a, b \geq 2}\left(\operatorname{tr} A_{a} A_{b}\right)^{2}=\sum_{a=2}^{p}\left(\operatorname{tr} A_{a}^{2}\right)^{2}$. Since $\operatorname{tr} A_{a}^{2}=\operatorname{tr} A_{f a}^{2}$ for $a \geq 2$, we have

$$
\sum_{a=2}^{p}\left(\operatorname{tr} A_{a}^{2}\right)^{2}=2 \sum_{a=2}^{p^{\prime}}\left(\operatorname{tr} A_{a}^{2}\right)^{2}=2\left(\left(\sum_{a=2}^{p^{\prime}} \operatorname{tr} A_{a}^{2}\right)^{2}-\sum_{a, b \geq 2, a \neq b}^{p^{\prime}} \operatorname{tr} A_{a}^{2} \operatorname{tr} A_{b}^{2}\right) .
$$

On the other hand, we see

$$
\left(\sum_{a=2}^{p} \operatorname{tr} A_{a}^{2}\right)^{2}=\left(2 \sum_{a=2}^{p^{\prime}} \operatorname{tr} A_{a}^{2}\right)^{2}=4\left(\sum_{a=2}^{p^{\prime}} \operatorname{tr} A_{a}^{2}\right)^{2} .
$$


Therefore

$$
\sum_{a=2}^{p}\left(\operatorname{tr} A_{a}^{2}\right)^{2}=\frac{1}{2}\left(\sum_{a=2}^{p} \operatorname{tr} A_{a}^{2}\right)^{2}-2 \sum_{a, b \geq 2, a \neq b}^{p^{\prime}} \operatorname{tr} A_{a}^{2} \operatorname{tr} A_{b}^{2} \leq \frac{1}{2}\left(\sum_{a=2}^{p} \operatorname{tr} A_{a}^{2}\right)^{2},
$$

from which $\sum_{a, b \geq 2}^{p}\left(\operatorname{tr} A_{a} A_{b}\right)^{2} \leq(1 / 2)\left(\sum_{a} \operatorname{tr} A_{f a}^{2}\right)^{2}$. Hence we have the second inequality.

Using Lemma 3-Lemma 8, we prove the following lemma.

LEMMA 9. Let $M$ be a compact $n$-dimensional minimal proper $C R$ submanifold of $C P^{m}$. If the Ricci tensor $S$ of $M$ satisfies $S(X, X) \geq(n-1) g(X, X)$, then $A_{f a}=0$ for all $a$.

PROOF. From Lemma 7 and Lemma 8, we have

$$
\begin{aligned}
& \frac{1}{2} \Delta\left(\sum_{a} \operatorname{tr} A_{f a}^{2}\right) \\
& \quad=\sum_{a \geq 2, i} g\left(\left(\nabla^{2} A\right)_{a} e_{i}, A_{a} e_{i}\right)+\sum_{a \geq 2, i} g\left((\nabla A)_{a} e_{i},(\nabla A)_{a} e_{i}\right) \\
& \quad \geq \sum_{a \geq 2, i} g\left(\left(\nabla^{2} A\right)_{a} e_{i}, A_{a} e_{i}\right) \\
& \quad=(n+4) \sum_{a} \operatorname{tr} A_{f a}^{2}-\sum_{a, b}\left|\left[A_{a}, A_{b}\right]\right|^{2}-\sum_{a, b \geq 2}\left(\operatorname{tr} A_{a} A_{b}\right)^{2} \\
& \quad \geq\left(\sum_{a} \operatorname{tr} A_{f a}^{2}\right)\left(n-\frac{1}{2} \sum_{a} \operatorname{tr} A_{f a}^{2}\right) .
\end{aligned}
$$

On the other hand, by the assumption, the Ricci tensor $S$ satisfies

$$
\sum_{i} S\left(e_{i}, e_{i}\right)=(n+3)(n-1)-|A|^{2} \geq(n-1) \sum_{i} g\left(e_{i}, e_{i}\right),
$$

which reduces to $|A|^{2}=\operatorname{tr} A_{v}^{2}+\sum_{a} \operatorname{tr} A_{f a}^{2} \leq 3(n-1)$. Moreover, Lemma 5 (b) implies $\operatorname{tr} A_{v}^{2} \geq n-1$. Hence we have $\sum_{a} \operatorname{tr} A_{f a}^{2} \leq 2(n-1)<2 n$. Therefore, by the Hopf's lemma, $\sum_{a} \operatorname{tr} A_{f a}^{2}$ is constant so that $\Delta\left(\sum_{a} \operatorname{tr} A_{f a}^{2}\right)=0$ (cf. [5; p. 338]). Thus we have $A_{f a}=0$ for all $a$.

(Proof of Theorem 2)

From Lemma 4 and Lemma 9, the first normal space of $M$ is of dimension 1 and parallel with respect to the normal connection. 
Let $S^{2 m+1}$ be a $(2 m+1)$-dimensional unit sphere. We consider the Hopf fibration $\pi$ : $S^{2 m+1} \rightarrow C P^{m}$. Then the first normal space of $\bar{M}=\pi^{-1}(M)$ in $S^{2 m+1}$ is of dimension 1 and is also parallel with respect to the normal connection. Therefore, there is a totally geodesic $(n+2)$-dimensional submanifold $S^{n+2}$ of $S^{2 m+1}$ containing $\bar{M}$ (cf. [4]). Hence there is a totally geodesic $C P^{(n+1) / 2}$ of $C P^{m}$ containing $M$ (cf. [15; p. 227]).

\section{Pinching theorems for the Ricci curvature}

To prove our theorems, we need some well-known results.

In the following, we take the unit normal vector field $v$ of a real hypersurface $M$ in $C P^{m}$, and we put $\xi=-J v$. Then $\xi$ is the unit tangent vector field of $M$ and $P^{2} X=-X+g(X, \xi) \xi$, $P \xi=0$. We also put $A_{v}=A$ to simplify the notation. Then $\nabla_{X} \xi=P A X$ for any vector field $X$ tangent to $M$.

PROPOSITION A([3]). Let $M$ be a real hypersurface (with unit normal vector $v$ ) of a complex projective space $C P^{m}$ on which $\xi$ is a principal curvature vector with principal curvature $\alpha=2 \cot 2 r$ and the focal map $\phi_{r}$ has constant rank on $M$. Then the following hold:

(a) $M$ lies on a tube (in the direction $\eta=\gamma^{\prime}(r)$, where $\gamma(r)=\exp _{x}(r v)$ and $x$ is a base point of the normal vector $v$ ) of radius $r$ over a certain Kähler submanifold $N$ in $C P^{m}$.

(b) Let $\cot \theta, 0<\theta<\pi$, be a principal curvature of the second fundamental form $A_{\eta}$ at $y=\gamma(r)$ of the Kähler submanifold $N$. Then the real hypersurface $M$ has a principal curvature $\cot (r-\theta)$ at $x=\gamma(0)$.

PROPOSITION $\mathrm{B}([10])$. Let $M$ be a real hypersurface of a complex projective space $C P^{m}$. If $A \xi=0$, except for the null set on which the focal map $\phi_{r}$ degenerates, $M$ is locally congruent to one of the following:

(a) a homogeneous real hypersurface which lies on a tube of radius $\pi / 4$ over a totally geodesic $C P^{k}(1 \leq k \leq m-1)$,

(b) a nonhomogeneous real hypersurface which lies on a tube of radius $\pi / 4$ over a Kähler submanifold $N$ with nonzero principal curvatures $\neq \pm 1$.

Using these results, we prove the following

THEOREM 3. Let $M$ be a compact $n$-dimensional minimal CR submanifold of a complex projective space $C P^{m}$ which is not a complex submanifold of $C P^{m}$. If the Ricci tensor $S$ of $M$ satisfies $S(X, X) \geq(n-1) g(X, X)$ for any vector field $X$ tangent to $M$, then $M$ is congruent to one of the following:

(a) a totally geodesic real projective space $R P^{n}$ of $C P^{m}$,

(b) a pseudo-Einstein real hypersurface $M^{c}((n-1) / 4, \pi / 4)$ of some $C P^{(n+1) / 2}$ in $C P^{m}$,

(c) a real hypersurface of some $C P^{(n+1) / 2}$ in $C P^{m}$ which lies on a tube of radius $\pi / 4$ over certain Kähler submanifold $N$ with principal curvatures $\cot \theta, 0<\theta \leq \pi / 12$. 
Proof. We suppose that $M$ is proper. Then Theorem 2 implies that $M$ is a real hypersurface of some totally geodesic complex projective space $C P^{(n+1) / 2}$ in $C P^{m}$. By the proof of Lemma 2, we have $A \xi=0$. On the other hand, from Lemma 5, we obtain $A P A X=P X$ for any $X$ tangent to $M$. Thus we see that if $A X=\lambda X$, then $A P X=(1 / \lambda) P X$. Since $3 g(P X, P X) \geq g\left(A^{2} X, X\right)$, we have $\lambda^{2} \leq 3$. We also have $\operatorname{rank} A \leq n-1$ because $A \xi=0$. A homogeneous real hypersurface which lies on a tube of radius $\pi / 4$ over a totally geodesic $C P^{k}$ is minimal if and only if $k=(n-1) / 4$, that is, $M$ is $M_{k, k}^{c}$. The principal curvatures of this real hypersurface is \pm 1 (see [3; p. 493]).

For a nonhomogeneous real hypersurface $M$ which lies on a tube of radius $\pi / 4$ over a Kähler submanifold $N$, by the condition $\lambda^{2} \leq 3$ and (b) of Proposition A, we see that $\cot ^{2}(\pi / 4-\theta) \leq 3$. Thus we have $0<\theta \leq \pi / 12$. Consequently, using Proposition $\mathrm{A}$ and Proposition $\mathrm{B}$, we have our theorem.

REMARK 1. The author does not know any example of a Kähler submanifold $N$ having the properties required in Case (c) in Theorem 3.

COROLLARY 1. Let $M$ be a compact $n$-dimensional minimal proper CR submanifold of a complex projective space $C P^{m}$. If the Ricci tensor $S$ of $M$ satisfies $S(X, X) \geq(n-$ 1) $g(X, X)$, then $M$ is congruent to one of the following:

(a) a pseudo-Einstein real hypersurface $M^{c}((n-1) / 4, \pi / 4)$ of some $C P^{(n+1) / 2}$ in $C P^{m}$,

(b) a real hypersurface of some $C P^{(n+1) / 2}$ in $C P^{m}$ which lies on a tube of radius $\pi / 4$ over certain Kähler submanifold $N$ with principal curvatures $\cot \theta, 0<\theta \leq \pi / 12$.

Using the theorem in [9], we have

COROLlARY 2. Let $M$ be a compact $n$-dimensional minimal proper CR submanifold of a complex projective space $C P^{m}, n \geq 5$. If the Ricci tensor $S$ satisfies $(n-1) g(X, X) \leq$ $S(X, X) \leq(n+1) g(X, X)$, then $M$ is congruent to a pseudo-Einstein real hypersurface $M^{c}((n-1) / 4, \pi / 4)$ of some $C P^{(n+1) / 2}$ in $C P^{m}$.

Next we prove the following

THEOREM 4. Let $M$ be a compact $n$-dimensional minimal $C R$ submanifold of a complex projective space $C P^{m}$. If the Ricci tensor $S$ of $M$ satisfies $S(X, X) \geq(n-1) g(X, X)+$ $g(P X, P X)$ for any vector field $X$ tangent to $M$, then $M$ is congruent to one of the following:

(a) a totally geodesic real projective space $R P^{n}$ of $C P^{m}$,

(b) a totally geodesic complex projective space $C P^{n / 2}$ of $C P^{m}$,

(c) a complex $(n / 2)$ dimensional complex quadric $Q^{(n / 2)}$ of some $C P^{n / 2+1}$ of $C P^{m}$,

(d) a pseudo-Einstein real hypersurface $M^{c}((n-1) / 4, \pi / 4)$ of some $C P^{(n+1) / 2}$ in $C P^{m}$,

(e) a real hypersurface of some $C P^{(n+1) / 2}$ in $C P^{m}$ which lies on a tube of radius $\pi / 4$ over certain Kähler submanifold $N$ with principal curvatures $\cot \theta$, where $\theta$ satisfies $0<\sin 2 \theta \leq 1 / 3$. 
For the proof of the theorem, we prepare some lemmas for complex submanifolds. We take an orthonormal basis $\left\{v_{1}, \ldots, v_{p}, v_{p+1}=f v_{1}, \ldots, v_{2 p}=f v_{p}\right\}$ of $T_{x}(M)^{\perp}$.

LEMMA 10 ([6]). Let $M$ be a complex k-dimensional Kähler submanifold of a complex $m$-dimensional Kähler manifold $\bar{M}$. Then

$$
\begin{gathered}
\frac{1}{k}|A|^{4} \leq \sum_{a, b=1}^{2 p}\left|\left[A_{a}, A_{b}\right]\right|^{2} \leq|A|^{4}, \\
\frac{1}{2 p}|A|^{4} \leq \sum_{a, b=1}^{2 p}\left(\operatorname{tr} A_{a} A_{b}\right)^{2} \leq \frac{1}{2}|A|^{4},
\end{gathered}
$$

where $p=m-k$. If $\bar{M}$ is of constant holomorphic sectional curvature $c$, then $M$ is Einstein if and only if $\sum_{a, b=1}^{2 p}\left|\left[A_{a}, A_{b}\right]\right|^{2}=|A|^{4} / k$.

From Theorem 1, we see

LEMMA 11. Let $M$ be a complex $k$-dimensional Kähler submanifold of $C P^{m}$. Then

$$
g\left(\nabla^{2} A, A\right)=2(k+2)|A|^{2}-\sum_{a, b=1}^{2 p}\left|\left[A_{a}, A_{b}\right]\right|^{2}-\sum_{a, b=1}^{2 p}\left(\operatorname{tr} A_{a} A_{b}\right)^{2} .
$$

In the following we prove Theorem 4. From Theorem 2, if $M$ is proper, then it is a real hypersurface of some $C P^{(n+1) / 2}$ in $C P^{m}$.

Next we suppose that $M$ is a complex $(n / 2)$ dimensional complex submanifold of $C P^{m}$. Since $M$ is complex minimal submanifold of $C P^{m}$, we have

$$
S(X, Y)=(n+2) g(X, Y)-\sum_{a=1}^{2 p} g\left(A_{a}^{2} X, Y\right) .
$$

Thus we have $\sum_{a=1}^{2 p} g\left(A_{a}^{2} X, X\right) \leq 2 g(X, X)$, from which $|A|^{2} \leq 2 n$. Moreover, we see that $2 I-\sum_{a} A_{a}^{2}$ is a positive semi-definite operator. The symmetricity of $A_{a}$ implies that $\sum_{a} A_{a}^{2}$ is positive semi-definite. The operators $\sum_{a} A_{a}^{2}$ and $2 I-\sum_{a} A_{a}^{2}$ can be transformed simultaneously by an orthogonal matrix into diagonal forms at each point of $M$, thus we see that $\left(\sum_{a} A_{a}^{2}\right)\left(2 I-\sum_{a} A_{a}^{2}\right)$ is positive semi-definite. Hence we have

$$
\operatorname{tr}\left(\sum_{a=1}^{2 p} A_{a}^{2}\right)^{2} \leq 2|A|^{2} \leq 4 n
$$

On the other hand, we obtain

$$
\sum_{a, b=1}^{2 p}\left|\left[A_{a}, A_{b}\right]\right|^{2}=2 \sum_{a, b=1}^{2 p} \operatorname{tr} A_{a}^{2} A_{b}^{2}=2 \operatorname{tr}\left(\sum_{a=1}^{2 p} A_{a}^{2}\right)^{2}
$$


Therefore we get $\sum_{a, b=1}^{2 p}\left|\left[A_{a}, A_{b}\right]\right|^{2} \leq 4|A|^{2}$. From Lemma 10, Lemma 11 and these equations, we have,

$$
\begin{aligned}
\frac{1}{2} \Delta|A|^{2} & =g\left(\nabla^{2} A, A\right)+|\nabla A|^{2} \\
& \geq g\left(\nabla^{2} A, A\right) \geq|A|^{2}\left(n-\frac{1}{2}|A|^{2}\right) \geq 0 .
\end{aligned}
$$

Hence, by the theorem of E. Hopf, $|A|^{2}$ is constant so that $\Delta|A|^{2}=0$ (cf. [5; p. 338]). Thus we have $|A|=0$ or $|A|^{2}=2 n$. When $|A|=0, M$ is totally geodesic.

Next we suppose $|A|^{2}=2 n$. By (5), we have $\operatorname{tr}\left(\sum_{a=1}^{2 p} A_{a}^{2}\right)^{2}=4 n$, that is,

$$
\sum_{a, b=1}^{2 p}\left|\left[A_{a}, A_{b}\right]\right|^{2}=8 n=\frac{2|A|^{4}}{n} .
$$

From Lemma 10, $M$ is an Einstein complex submanifold of $C P^{m}$.

For any normal vector field $V$ with $V_{x} \in N_{0}(x)=\left\{V \in T_{x}(M)^{\perp}: A_{V}=0\right\}$, we have

$$
\nabla_{Y}\left(A_{V} X\right)=\left(\nabla_{Y} A\right)_{V} X+A_{D_{Y} V} X+A_{V}\left(\nabla_{Y} X\right)=0
$$

at $x \in M$. Hence $A_{D_{Y} V} X+\left(\nabla_{Y} A\right)_{V} X=0$. Since the equality of (6) holds, we get $\nabla A=0$, from which we see that $N_{0}$ is parallel with respect to the normal connection. Let $V \in N_{0}$ and $U \in N_{1}$. Then

$$
X g(U, V)=g\left(D_{X} U, V\right)+g\left(U, D_{X} V\right)=0 .
$$

Hence the first normal space is parallel with respect to the normal connection. On the other hand, since the equality of (6) holds, we have $\sum_{a, b=1}^{2 p}\left(\operatorname{tr} A_{a} A_{b}\right)^{2}=(1 / 2)|A|^{4}$. In the next place, we take a basis $\left\{v_{1}, \ldots, v_{p}, v_{p+1}=f v_{1}, \ldots, v_{2 p}=f v_{p}\right\}$ of $T_{x}(M)^{\perp}$ such that $\sum_{a, b=1}^{2 p}\left(\operatorname{tr} A_{a} A_{b}\right)^{2}=\sum_{a=1}^{2 p}\left(\operatorname{tr} A_{a}^{2}\right)^{2}$. Then

$$
\sum_{a=1}^{2 p}\left(\operatorname{tr} A_{a}^{2}\right)^{2}=\frac{1}{2}|A|^{4}-2 \sum_{a \neq b}^{p}\left(\operatorname{tr} A_{a}^{2}\right)\left(\operatorname{tr} A_{b}^{2}\right),
$$

and therefore $\sum_{a \neq b}^{p}\left(\operatorname{tr} A_{a}^{2}\right)\left(\operatorname{tr} A_{b}^{2}\right)=0$. This implies $\operatorname{dim} N_{1}=2$. Consequently, $M$ is an Einstein complex hypersurface of some $C P^{n / 2+1}$ in $C P^{m}$, that is, a complex quadric $Q^{n / 2}$ of $C P^{n / 2+1}$ (see [13]). From this and Theorem 3, we have our theorem.

REMARK 2. In 1974, Chen and Ogiue [2] proved that if the Ricci curvature of $n$ dimensional Kähler submanifold of $C P^{m}$ is everywhere equal to $n / 2$, then $M$ is locally $Q^{n}$ in some $C P^{n+1}$ in $C P^{m}$ (see also [11]). 
We suppose that $M$ is a compact $n$-dimensional minimal $C R$ submanifold of a complex projective space $C P^{m}$. When the Ricci tensor $S$ of $M$ satisfies $S(X, X) \geq(n-1) g(X, X)+$ $2 g(P X, P X)$ for any vector $X$ tangent to $M$, the cases (c) and (e) in Theorem 4 do not occur. Thus we obtain

THEOREM 5 ([8]). Let $M$ be a compact $n$-dimensional minimal $C R$ submanifold of a complex projective space $C P^{m}$. If the Ricci tensor $S$ of $M$ satisfies $S(X, X) \geq(n-$ 1) $g(X, X)+2 g(P X, P X)$ for any vector field $X$ tangent to $M$, then $M$ is congruent to one of the following:

(a) a totally geodesic real projective space $R P^{n}$ of $C P^{m}$,

(b) a totally geodesic complex projective space $C P^{n / 2}$ of $C P^{m}$,

(c) a pseudo-Einstein real hypersurface $M^{c}((n-1) / 4, \pi / 4)$ of some $C P^{(n+1) / 2}$ in $C P^{m}$.

\section{References}

[ 1] K. ABE, Applications of Riccati type differential equation to Riemannian manifolds with totally geodesic distribution, Tôhoku Math. J. 25 (1973), 425-444.

[ 2 ] B-Y. Chen and K. Ogiue, A characterization of the complex sphere, Michigan Math. J. 21 (1974), $231-232$.

[ 3 ] T. E. CECIL and P. J. RYAN, Focal sets and real hypersurfaces in complex projective space, Trans. Amer. Math. Soc. 269 (1982), 481-499.

[ 4 ] J. ERBACHER, Reduction of the codimension of an isometric immersion, J.Differential Geom. 5 (1971), 333340.

[ 5 ] S. Kobayashi and K. NomizU, Foundations of differential geometry, Vol. II, Interscience, New York, 1969.

[ 6 ] MASAHIRo Kon, On some complex submanifolds in Kaehler manifolds, Canad. J. Math. 26 (1974), 14421449.

[ 7 ] Masahiro Kon, Generic minimal submanifolds of a complex projective space, Bull. London Math. Soc. 12 (1980), 355-360.

[ 8 ] MASAHIRo Kon, Minimal $C R$ submanifolds immersed in a complex projective space, Geom. Dedicata 31 (1989), 357-368.

[ 9 ] S. MaedA, Real hypersurfaces of a complex projective space II, Bull. Austral. Math. Soc. 29 (1984), $123-127$.

[10] S. MAEDA, Ricci tensors of real hypersurfaces in a complex projective space, Proc. Amer. Math. Soc. 122 (1994), 1229-1235.

[11] K. OgIUE, Differential geometry of Kaehler submanifolds, Advances in Math. 13 (1974), $73-114$.

[12] J. Simons, Minimal varieties in riemannian manifolds, Ann. of Math. 88 (1968), 62-105.

[13] B. Smith, Differential geometry of complex hypersurfaces, Ann. of Math. 85 (1967), 246-266.

[14] M. YAMAGATA and MASAHIRO KON, Reduction of the codimension of a generic minimal submanifold immersed in a complex projective space, Coll. Math. 74 (1997), 185-190.

[15] K. YANO and MASAhIRo Kon, Structures on manifolds, World Scientific Publishing, Singapore, 1984.

Present Address:

FACULTY OF EDUCATION,

SHINSHU UNIVERSITY,

6-Ro, NishinAGANO, NAGANO CITY, 380-8544 JAPAN.

e-mail: mayuko_k@shinshu-u.ac.jp 